\title{
Active Self-Interference Cancellation Mechanism for Full-Duplex Wireless Transceivers
}

\author{
Ramez Askar and Thomas Kaiser \\ Institute of Digital Signal Processing \\ Faculty of Engineering \\ University of Duisburg-Essen \\ D-47057 Duisburg, Germany
}

\author{
Benjamin Schubert, Thomas Haustein and Wilhelm Keusgen \\ Dept. of Wireless Communications and Networks \\ Fraunhofer Institute for Telecommunications, \\ Heinrich-Hertz-Institute \\ D-10587 Berlin, Germany
}

\begin{abstract}
This paper investigates active cancellation techniques for a Full-Duplex transceiver. An auxiliary transmit chain is employed to create the cancellation signal, which is then injected at the receiver $R F$ front-end by using a microstrip coupler. Two methods are proposed to calculate the self-interference cancellation signal. While the first method assumes the transmit chain to be strictly linear, the second one additionally incorporates nonlinear effects, occurring especially in the RF power amplifier. The linear method reports experimentally around $48 \mathrm{~dB}$ of selfinterference suppression under linear system behavior. Experimental results show also $50 \mathrm{~dB}$ of suppression by using the nonlinear method and under nonlinear system behavior, whereas the linear one reports $47 \mathrm{~dB}$ of suppression under the same conditions.
\end{abstract}

\section{INTRODUCTION AND THE STATE OF THE ART}

The increasing demand for higher data rates and service proliferation everywhere at any time to everybody makes the better use of wireless resources a prerequisite. Schemes to optimize radio resources in time, frequency and space are well understood for years; nevertheless, state-of-the-art wireless transmission schemes are suffering from a specific limitation in resource utilization namely simultaneous transmission and reception on the same frequency and time resource. Current state of the art solutions are either time division duplex (TDD e.g. WiFi, LTE-TDD) or frequency division duplex (FDD e.g. GSM, UMTS, LTE-FDD) schemes. Such systems are called half-duplex since transmission and reception are not performed at the same time and frequency. In contrast, a full-duplex system is able to choose uplink and downlink or forward and reverse link frequencies more flexible and preferably without restrictions. Full-Duplex Enabling Technology will be a decisive contribution to achieve more flexible and improved spectrum utilization.

The key challenge in realizing a full-duplex system is the self-interference signal which can be $100 \mathrm{~dB}$ stronger than the desired receive signal; due to the proximity between transmit and receive antenna at the full-duplex node. Hence, signal reception becomes impossible unless the self-interference is dramatically suppressed.

Many self-interference cancellation techniques have been discussed in the literature. For instance [1] and [2] proposed a cancellation technique based on a specific antenna placement. This technique requires two transmit antennas spaced apart from the receiving antenna by the distances $d$ and $d+\frac{\lambda}{2}$, respectively. In that way, the two transmit antennas will superpose a null at the receive antenna location. Despite the high accuracy requirements in the antenna placement process, this technique is only viable in a narrow-band scenario and will fail in case of broadband signals.

In [3] further work has been done, whereas the cancellation signal is generated by means of a RF Balun instead of the additional antenna element. The required delay and attenuation for the cancellation signal are applied with the aid of a noise canceling integrated circuit. The results of the RF Balun implementation are much better from the earlier work with antenna placement, particularly in broadening the selfinterference cancellation bandwidth. However, this approach is still falling short of the self-interference cancellation requirements even with a consecutive stage of digital self-interference cancellation. Additionally there are some practical implications like additional nonlinearities from the noise canceling circuit and imperfections of the RF balun.

In [4] and [5], other antenna-related passive cancellation techniques have been investigated. These techniques rely on the antenna directivity in combination with other methods concerning for instance the physical separation of the antennas, different polarizations and additional RF absorbing materials. One main drawback of these canceling techniques is that the uplink and downlink of the full-duplex node are spatially separated and they are directed towards two different locations. This will make such a technique not applicable in the point-topoint scenarios where both communication nodes are operating in full-duplex mode.

Recently, researchers at the Stanford University reported promising results for full-duplex communication systems [6], [7]. In certain use case scenarios the full-duplex system achieved a twice as high spectral efficiency as the half-duplex system. This cancellation technique is based on a printed circuit board with multiple routes, all equipped with adjustable attenuators and all having a different length in order to provide several delays. The entire design has been used to reproduce the circulator leakage and the antenna mismatch reflection. Regardless of the contribution of the Stanford team to proof the concept, many other considerations have to be taken into account in the commodity wireless hardware like having relatively nearby obstacles around the full-duplex node.

Another self-interference cancellation technique has been proposed by the scientists at Rice University [8], [9]. This technique has been characterized in the literature as active 


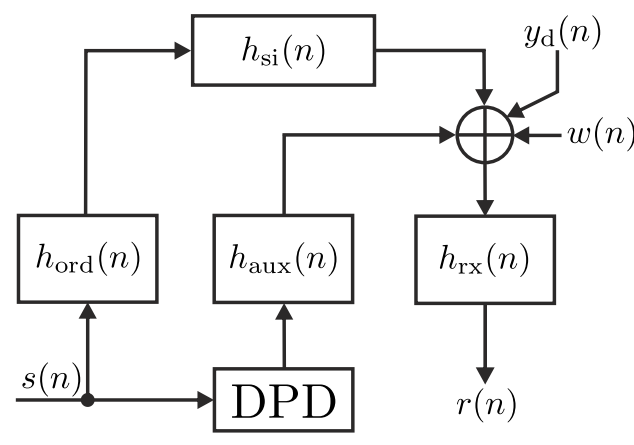

Fig. 1. The system model of a full-duplex transceiver utilizing the active cancellation method to cancel the self-interference.

cancellation technique due to its mechanism in which the fullduplex transceiver requires an additional transmission chain. This additional chain actively injects a replica of the selfinterference signal with a negative magnitude at the receiver $\mathrm{RF}$ front-end of the full-duplex node.

The work presented here adopts the active self-interference cancellation mechanism similar to the approach in [8]. A single antenna experiment with a RF circulator approach has been conducted. Moreover, a linear and a nonlinear digital predistortion model have been developed. These pre-distortion models have been implemented and tested on an experimental testbed. The required self-interference suppression amount has been analyzed in accordance to a real-world transceiver design. Furthermore, the impact of the impairments of the real-world transceivers on the self-interference active cancellation method has been characterized.

The rest of this paper is organized as follows. Section II provides the signal and system model of the proposed fullduplex wireless transceiver concept and introduces the active self-interference cancellation mechanism. In Section III the requirements for self-interference cancellation in a full-duplex transceiver are specified. Section IV studies two models of digital pre-distortion. Section V describes the experimental setup and reports the experimental results. Finally, the conclusions are drawn in Section VI.

Notation: $\mathcal{F}, \mathcal{F}^{-1}$ and $*$ denotes Fourier transform, inverse Fourier transform and convolution, respectively.

\section{TRANSCEIVER CONCEPT AND MECHANISM}

Basically, the structure of the full-duplex transceiver consists of three essential chains. The first chain is the ordinary transmission chain, this chain is identical to the one you find in a conventional half-duplex transceiver. The transmission through this chain, which is designated to be received at the remote node, is also received by the node itself and causing the self-interference. The second chain is the auxiliary transmission chain. This chain from the structure point of view is identical to the ordinary one, only with less power amplification. The purpose of this chain is to realize the active self-interference cancellation mechanism in the fullduplex transceiver. The auxiliary transmission chain is dedicated to replicate a version of the self-interference signal with a negative magnitude and injected at the receiver RF frontend of the full-duplex transceiver. The auxiliary chain has a hybrid structure which spreads over two domains, the digital and the analog one. Practical aspects have to be considered for the design of such a structure. The digital domain access allows to replicate any discrepancies between the ordinary and the auxiliary one, particularly the self-interference wireless channel. The analog domain access allows us to avoid saturating the receiving chain by injecting the cancellation signal before the low noise amplification stage. The last chain is the receiving chain where the desired reception signal corrupted with residual self-interference is received and down-converted to the digital domain.

\section{A. Transceiver signal and system model}

Fig. 1 depicts the system model of the full-duplex transceiver adopting the active cancellation mechanism. $h_{\text {ord }}(n)$ represents the equivalent baseband channel between the generated waveform from the digital to analog convertor (DAC) and the RF power amplifier (PA) output at the ordinary transmission chain. $h_{\text {aux }}(n)$ represents the equivalent baseband channel between the generated waveform from DAC and the PA output at the auxiliary transmission chain. $h_{\mathrm{si}}(n)$ represents the equivalent baseband channel impulse response of the selfinterference radio channel. $h_{\mathrm{rx}}(n)$ represents the equivalent baseband channel of the receiving chain at the transceiver. $s(n)$ and $r(n)$ are the transmitted and the received complex baseband samples, respectively. The received baseband signal composes of the desired reception signal $y_{\mathrm{d}(n)}$, the residual self-interference $r_{\mathrm{rsi}(n)}$ and the multiple source noise $w_{\mathrm{r}}(n)$,

$$
r(n)=r_{\mathrm{d}}(n)+r_{\mathrm{rsi}}(n)+w_{\mathrm{r}}(n),
$$

where $r_{\mathrm{d}}(n)=y_{\mathrm{d}(n)} * h_{\mathrm{rx}}(n)$.

For simplicity, let us assume that the ordinary and the auxiliary chains are identical ${ }^{1}$, therefore, their channel models are equal $h_{\text {ord }}(n)=h_{\text {aux }}(n)$. Thus, the digital per-distorter (DPD) needs to be equipped with estimated self-interference wireless channel impulse response $\tilde{h}_{\mathrm{si}}(n)$. The residual selfinterference in its baseband form is then given by

$$
r_{\mathrm{rsi}}(n)=\left(h_{\mathrm{si}}(n)-\tilde{h}_{\mathrm{si}}(n)\right) * h_{\mathrm{rx}}(n) * h_{\mathrm{ord}}(n) * s(n) .
$$

\section{B. Self-interference cancellation mechanism}

The active self-interference cancellation mechanism in the full-duplex transceiver has to major phases. The full-duplex transceiver must pass these phases successfully in order to start operating in a full-duplex mode. A tracking phase would be necessary to keep the full-duplex communication scheme alive on the transceiver. The tracking algorithm assures that the predistorter parameters remain up-to-date.

1) Parameter acquisition phase: Or the initial phase for the full-duplex transceiver. During this phase, the full-duplex transceiver obtains all the parameters which are required for the next operation phase. The parameters are estimated sequentially; starting from the most stationary parameter and ending up with the most variable one. During this phase the transceiver is not ready yet to operate in a full-duplex mode. A training-based pilot signal is used to estimate the required channels. The obtained parameters are stored in the transceiver and prepared to be used in the next phase.

\footnotetext{
${ }^{1}$ In practice such assumption is not realistic but it is considered to generate a mathematically friendly model clarifying the DPD operational role
} 
2) Pre-distorter construction phase: The digital predistorter (DPD) is the key element in enabling a broadband full-duplex wireless communication scheme. All acquired parameters are utilized to build up a pre-distortion model. The constructed pre-distortion model is placed in the digital domain at the beginning of the auxiliary chain. A copy of the ordinary transmission signal in its baseband form goes through the DPD in order to be prepared for the cancellation process. A perfect DPD pre-distorts the baseband to be precisely identical to the self interference signal at the receiver front-end, however, with negative amplitude. In that way, the self-interference can be eliminated totally. Two DPD models have been developed and tested in this work, see section IV. A substantial part has to be included in any constructed pre-distortion model which is the multipath self-interference wireless channel. Additionally, any source of discrepancies between the ordinary and auxiliary chains can be included in the pre-distorter design in order to improve its cancellation performance. The tradeoff between DPD cancellation performance, on the one hand, and the DPD model complexity, on the other, has to be considered in its design constrains. The algorithm execution time which is composed of the acquisition and construction phases has to be shorter than the coherence time of the self-interference wireless channel; under the assumption that the wireless channel is the fastest changing part in the applied DPD model.

3) Adaptive pre-distorter and tracking phase: The assignment of the former phases is to attain a sufficient amount of self-interference suppression which enables a basic communication with the other node in full-duplex scheme. While, the assignment of the tracking phase is to maintain this level of self-interference suppression under a certain threshold in order to avoid saturating at the receiving chain in the full-duplex transceiver. The saturation threshold can be determined from the transceiver hardware specifications, which is the full scale power level of the analog to digital convertor (ADC) in realworld transceiver designs. The tracking algorithm observes the deterioration in self-interference cancellation performance and triggers the estimation phase again whenever it exceeds the specified saturation threshold. The changes in the surrounding environment in addition to the full-duplex node mobility conditions play decisive roles in determining the expiration period of the constructed DPD model.

\section{SELF-INTERFERENCE CANCELLATION REQUIREMENTS}

The performance of a full-duplex system is characterized by the amount of self-interference cancellation which its transceiver can achieve. A fully operational full-duplex transceiver is able to bring the self-interference down to the receiver noise floor $\left(\mathrm{NF}_{\mathrm{R}}\right)$ level, doubling by that the throughput of the wireless communication system. To provide a deeper insight into the self-interference cancellation (SIC) requirements and its relation to the transceiver specifications, the power levels for a full-duplex transceiver are illustrated in Fig. 2. $P_{\mathrm{T}}$ is the transmission power of the wireless transceiver which is equivalent to the self-interference power level in a full-duplex transceiver. $P_{\mathrm{R}}$ is the power level of the desired reception signal coming from the remote communication node. FS is the full scale level of the ADC in receiving chain which draws the upper bound where $P_{\mathrm{R}}$ must lay under it; in avoidance of any signal clipping. DR is the dynamic range of the ADC which is

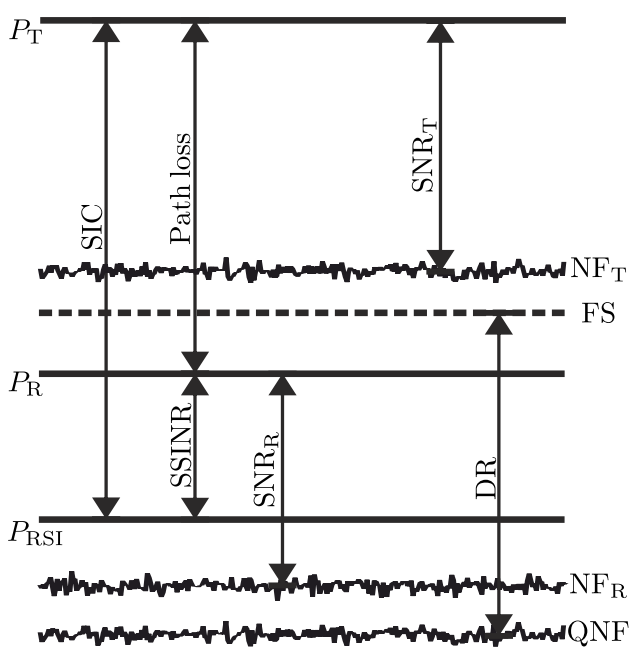

Fig. 2. Power level chart illustrates the dependencies between the fullduplex transceiver specifications and the achievable amount of self-interference cancellation.

determined by the resolution of the convertor. The path loss in Fig. 2 belongs to the wireless channel between the local and remote node. On the first hand and assuming that the remote transceiver has a transmission power $P_{\mathrm{T}}$ as much as the local one, the received signal power is then given by

$$
P_{\mathrm{RdBm}}=P_{\mathrm{TdBm}}-\text { Path } \operatorname{loss}_{\mathrm{dB}} .
$$

On the other hand, the residual self-interference after the selfinterference cancellation process can be expressed as

$$
P_{\mathrm{RSIdBm}}=P_{\mathrm{TdBm}}-\mathrm{SIC}_{\mathrm{dB}} .
$$

Combining (3) and (4), the signal-to-self-interference-plusnoise-ratio (SSINR) in a full-duplex wireless transceiver can be expressed by

$$
\begin{aligned}
\mathrm{SSINR}_{\mathrm{dB}} & =P_{\mathrm{RdBm}}-P_{\mathrm{RSIdBm}} \\
& =- \text { Path loss } \\
\mathrm{dB} & +\mathrm{SIC}_{\mathrm{dB}} .
\end{aligned}
$$

The SSINR won't have a positive value unless the SIC is greater than the wireless channel path loss. Therefore, we can conclude that the achievable amount of the self-interference cancellation determines the affordable distance which could split two full-duplex nodes in a point-to-point full-duplex scenario.

Another factor limits the self-interference cancellation performance, particularly the active cancellation method, which is the transmitter noise $\mathrm{NF}_{\mathrm{T}}$ [10]. The auxiliary transmission chain in the active cancellation mechanism is not able to model the noise in the baseband because it is noise by definition, thus, the active cancellation method is not capable of canceling the transmitter noise. Consequently, a passive cancellation stage is necessary here in order to suppress the transmitter generated noise and besides that also reduces the active cancellation requirements.

In practice, the residual self-interference $P_{\mathrm{RSI}}$ in a fullduplex transceiver is higher than the receiver noise floor $N_{R}$. Fig. 2 illustrates the deterioration in the system SNR which occurs due to the residual self-interference dominating level. Accordingly, a full-duplex system has lower SNR levels, 
TABLE I. PILOT SIGNAL PARAMETERS

\begin{tabular}{c|c} 
Pilot parameter & Parameter value \\
\hline \hline Bandwidth & $20 \mathrm{MHz}$ \\
Number of subcarriers & 512 \\
Number of null subcarriers & 96 \\
Number of active subcarriers & 416 \\
Cyclic prefix length & 16 samples \\
& $800 \mathrm{~ns}$ \\
Subcarriers spacing & $39.063 \mathrm{kHz}$ \\
Number of repetition & 100
\end{tabular}

indicated here as SSINR, in comparison to the receiver $\mathrm{SNR}_{R}$ in a half-duplex one. The full-duplex transceiver design reaches its ultimate goal only if the proposed design of the transceiver is capable of bringing the residual self-interference $P_{\mathrm{RSI}}$ level down to the receiver noise floor $\mathrm{NF}_{\mathrm{R}}$.

\section{DIGITAL PRE-DISTORTER}

The digital pre-distorter (DPD) is created based on the estimated parameters, as we mentioned earlier in section II-B. Afterwords, this pre-distorter is used to cancel the selfinterference actively alongside the normal data transmission period. Therefore, the assumption that the self-interference wireless channel is stationary has to be hold in order to assure that the constructed model of pre-distortion remains valid to cancel the self-interference during the following data transmission phase.

In this paper, two DPD models are presented and tested experimentally. The acquisition and the construction phases have been covered, while, the tracking phase has been left for future work with real-time implementation.

\section{A. Linear model of pre-distortion}

In order to construct a linear pre-distorter (LPD), three consecutive steps have to be accomplished successfully. The first two steps belong to the acquisition phase: First, an OFDM based pilot signal goes through the ordinary chain and the receiver chain, allowing for the estimation of the complete transceiver in addition to the self-interference wireless channel. In the second step of the acquisition phase, the same pilot signal estimates the complete auxiliary chain including the receiving chain. Table I summarizes the main parameters of the pilot signal that has been used in this work.

The last step is the construction phase. During this step the LPD is computed to be

$$
g_{\mathrm{LPD}}(n)=h_{\mathrm{ord}}(n) * h_{\mathrm{si}}(n) * \mathcal{F}^{-1}\left\{\frac{1}{\mathcal{F}\left\{h_{\mathrm{aux}}(n)\right\}}\right\} .
$$

\section{B. Nonlinear model of pre-distortion}

The nonlinear pre-distorter (NLPD) is an extended model from the linear one. This extension in the pre-distortion model is meant to handle the nonlinearities which are introduced to the self-interference signal from the full-duplex transceiver components. In order to estimate these nonlinearities a Volterra series has been employed. The Volterra series has a mathematical form

$$
r(n)=\sum_{k=1}^{K} \sum_{n_{1}=0}^{N_{k}-1} \cdots \sum_{n_{k}=0}^{N_{k}-1} h_{k}\left(n_{1}, \ldots, n_{k}\right) \cdot \prod_{p=1}^{k} s\left(n-n_{p}\right),
$$

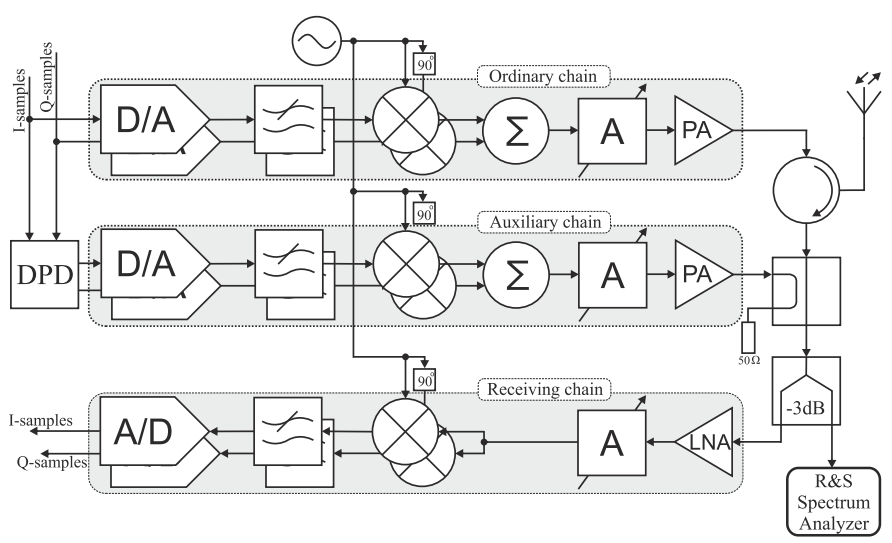

Fig. 3. The experimental setup, the $3 \mathrm{~dB}$ power splitter right after the microstrip coupler is placed to measure the residual self-interference signal at the RF domain by means of the spectrum analyzer.

where $s(n)$ and $r(n)$ are the transmitted and the received signals, respectively. The coefficients of the Volterra series $h_{k}\left(n_{1}, \ldots, n_{k}\right)$ have been identified using a method described in [11]. The identified nonlinear Volterra model of the ordinary chain is plugged to the LPD earlier model to yield the nonlinear one.

Note that, the nonlinear model of pre-distortion is able to cope with nonlinear transceiver behavior. Therefore, the nonlinear model is expected to have a better self-interference suppression performance, especially with increasing order of nonlinearities. However, it is obvious that its construction time takes much longer than the linear one which limits its performance with shorter coherence time of the self-interference wireless channel.

\section{SINGLE ANTENNA FULL-DUPLEX EXPERIMENT}

\section{A. Experimental setup}

In order to evaluate the efficiency of the active cancellation mechanism, an experimental setup has been prepared. The HIRATE platform [12] has been utilized to build the structure of the full-duplex transceiver working at $2.4 \mathrm{GHz}$. The experimental setup comprises of the following chains: The ordinary transmission chain which is connected to the receiving chain and the antenna via 3-port RF circulator [13], the auxiliary transmission chain which its RF output signal coupled to receiving chain front-end with aid of 4-port microstrip directional coupler and the receiving chain. Fig. 3 shows the structure of the experimentation testbed. This experimental setup is based on the conceptual structure of the full-duplex transceiver with active self-interference cancellation mechanism which has been descried previously in section II. The RF circulator has been used in the full-duplex transceiver design to benefit from its attenuation over the self-interference radio channel, moreover, to reduce the number of required antennas from two dedicated send and receive antennas to one single antenna. For the transceiver font-ends, direct up-conversion modulators and a direct down-conversion demodulator are used in the transmission chains and the receiving one, respectively. 


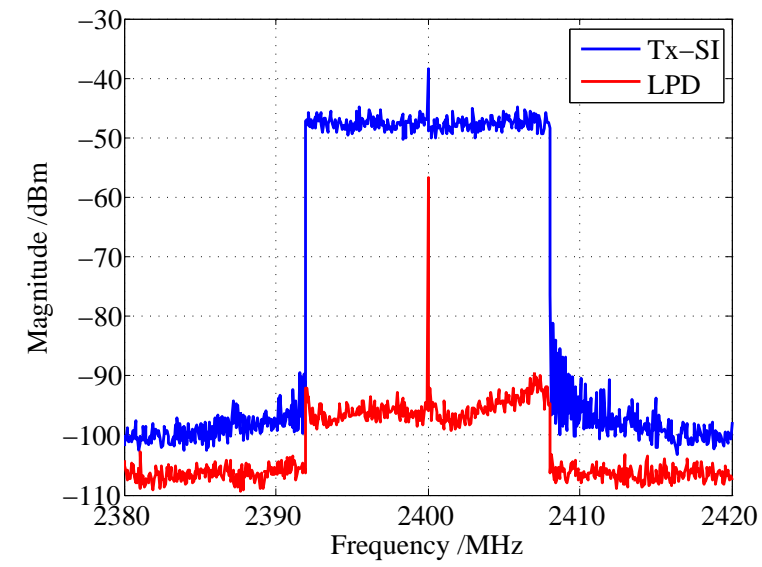

Fig. 4. Spectrum snapshots have been taken from the R\&S ${ }^{\circledR}$ FSU67 spectrum analyzer. The blue curve is measured at the ordinary chain RF output right after the PA, whereas the red curve is measured at the receiving chain front-end before the LNA as shown in Fig. 3.

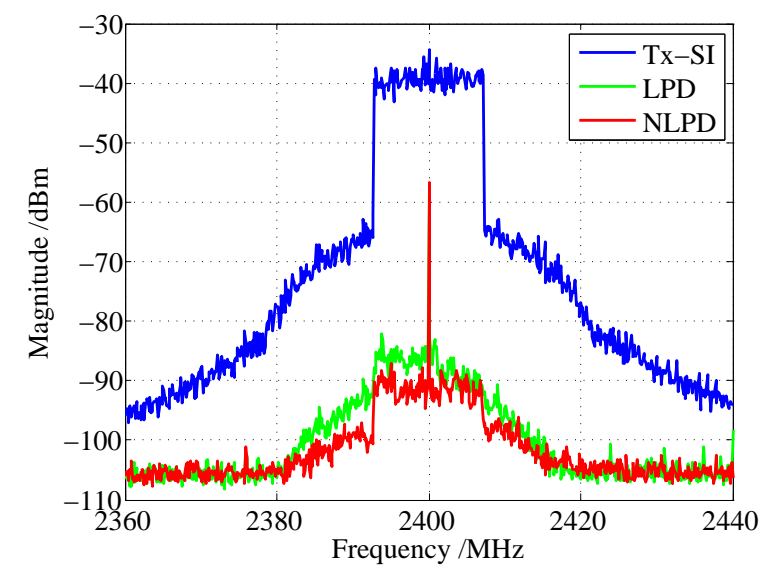

Fig. 5. Spectrum snapshots have been taken from the R\&S ${ }^{\circledR}$ FSU67 spectrum analyzer. The blue curve represents the ordinary chain RF output signal measured before it enters the circulator. The green and the red curves are measured at the receiving chain front-end before the LNA as shown in Fig. 3.

\section{B. Experiment procedure}

The digital signal processing is done offline in MATLAB ${ }^{\circledR}$. The I/Q samples of the digital baseband are prepared in MATLAB ${ }^{\circledR}$, afterward, these samples are sent to the HIRATE platform to be transmitted through the transceiver chains. The other way around is considered for the received signals. The received I/Q samples are temporarily stored in the HIRATE memory and send to the MATLAB ${ }^{\circledR}$ workspace afterward.

A couple of experiments has been conducted at Fraunhofer Heinrich-Hertz-Institute laboratories to test the efficiency of the proposed pre-distortion models. The first experiment aims to test the active cancellation mechanism in a full-duplex transceiver with the proposed linear model of pre-distortion, LPD. During this experiment, the transceiver components are kept working at the linear region, thus, the LPD model can be tested under linear system behavior; without any nonlinearity introduced from the transceiver components. The experiment procedure starts with transmitting I/Q samples randomly generated in the baseband through the ordinary chain. The spectrum of these samples are captured at the ordinary transmitter frontend by means of a spectrum analyzer, the spectrum is plotted with blue color in Fig. 4. In the second step of the experiment procedure, the LPD linear function is computed based on the estimated parameters of the transceiver chains plus the selfinterference wireless channel. For estimation purpose, a pilot signal goes through the ordinary and the auxiliary chain and is received by the receiving chain. At this stage, the efficiency of the active cancellation method with the LPD model can be tested, therefore, the I/Q samples generated earlier are transmitted through the ordinary chain alongside with a predistorted version through the auxiliary chain. The residual selfinterference signal is measured at the receiver RF front-end by using a spectrum analyzer as depicted in the experimental setup, see Fig. 3. The spectrum of the residual self-interference signal is plotted with red color in Fig. 4.

In order to test the efficiency of the NLPD model, a nonlinear system behavior had to be generated. Therefore, the PA in the ordinary chain was extended to a cascade configuration by attaching a second PA after the first one. In this way the first PA drives the second one to work in the nonlinear region. To avoid saturating the receiving chain an attenuator has been placed right after the cascade PAs; only for experimentation purpose. This altered experimental setup has been used to test the performance of both pre-distortion models. Hence, the efficiency of both LPD and NLPD in terms of self-interference suppression are tested under nonlinear system behavior. The same experiment procedure has been repeated as in the first experiment for the second and the third experiments in order to test NLPD and LPD, respectively. Note that, the parameters acquisition and pre-distortion construction phases are extended in the second experiment in order to compute the NLPD model. The same I/Q random samples are used to identify the coefficients of the Volterra series. The measured spectrums of the self-interference signal and the residual self-interference signals resulting from NLPD and LPD are all plotted in Fig. 5.

\section{Experiment results}

Fig. 4 shows the results from the first conducted experiment. In this experiment the LPD has been implemented and tested under linear system behavior. In comparison between the blue and the red curve, the LPD model reports around $48 \mathrm{~dB}$ of self-interference suppression under linear system behavior. Only around $28 \mathrm{~dB}$ of the overall suppression is resulting from the active cancellation method and the rest is done passively by the RF circulator.

The results of two conducted experiments under nonlinear system behavior are shown in Fig. 5. The blue spectrum represents the self-interference signal measured at the ordinary transmitter front-end. The regrowth of the shoulders around the in-band transmission signal indicates the nonlinear effects which caused by the cascade PAs configuration. In the same figure, the green spectrum and the red spectrum represent the residual self-interference signals which are measured at the receiver front-end. The second experiment tests the NLPD performance in terms of suppressing the self-interference under nonlinear system behavior; the result is illustrated with red curve in Fig. 5. In the third experiment, the self-interference suppression performance of the LPD model is tested under 
nonlinear system behavior; the spectrum of the residual selfinterference is illustrated with green curve in Fig. 5. Based on the experimental results, the LPD model reported around $47 \mathrm{~dB}$ of self-interference suppression, whereas the NLPD model reported more than $50 \mathrm{~dB}$ of self-interference suppression. The suppression of the nonlinear shoulders explains the difference in cancellation capabilities of the LPD and NLPD models. The LPD model suppressed the shoulders by $24 \mathrm{~dB}$, this suppression is solely a passive suppression due to the circulator leakage and the power splitter. However, the NLPD model suppressed the shoulder around $8 \mathrm{~dB}$ more than the LPD one, this extra suppression was apparently an active one and express the effectiveness of the NLPD in canceling the nonlinearities.

\section{CONCLUSIONS}

In this paper, the concept of a full-duplex wireless transceiver with active self-interference cancellation mechanism has been studied. Two models of pre-distortion, linear and nonlinear, have been developed. A single antenna fullduplex experiment with an RF circulator approach has been built. The pre-distortion models have been tested on the experimental setup. The experiments have reported self-interference suppression range between $45 \mathrm{~dB}$ and $50 \mathrm{~dB}$, with this limited amount of suppression the full-duplex scheme can be used only for short range communications. Recall from section III the power level chart, and assuming Wi-Fi transceiver has transmission power $20 \mathrm{dBm}$ and noise floor around $-90 \mathrm{dBm}$, thereby $110 \mathrm{~dB}$ of self-interference suppression are required to enable an ideal full-duplex scheme over the transceiver. By comparing the $110 \mathrm{~dB}$ to the experimental results, the active cancellation method with circulator approach falls short from its optimum goal.

Obviously, the active cancellation mechanism relies substantially on the precision of cancellation signal, therefore, the hardware impairments like the I/Q imbalance or the phase noise; which has been characterized in the literature as the the bottleneck of the active cancellation method [14], limit the cancellation performance. Accordingly, the current research focuses the effort on two major points: Building a comprehensive model of pre-distortion incorporating different hardware impairments like the I/Q imbalance, and enhancing the transceiver design to be able to cope with some impairments which are difficult to compensate in the baseband like the transmitter noise.

\section{ACKNOWLEDGMENT}

This work has been done within a cooperation framework between the department of Wireless Communications and Net- works at Fraunhofer Heinrich-Hertz-Institute and the Institute of Digital Signal Processing at University of Duisburg-Essen.

\section{REFERENCES}

[1] J. I. Choi, M. Jain, K. Srinivasan, P. Levis, and S. Katti, "Achieving single channel, full duplex wireless communication," in Proceedings of the sixteenth annual international conference on Mobile computing and networking, ser. MobiCom '10. New York, NY, USA: ACM, 2010, pp. $1-12$.

[2] M. A. Khojastepour, K. Sundaresan, S. Rangarajan, X. Zhang, and S. Barghi, "The case for antenna cancellation for scalable full-duplex wireless communications," in Proceedings of the 10th ACM Workshop on Hot Topics in Networks, ser. HotNets-X. New York, NY, USA: ACM, 2011, pp. 17:1-17:6.

[3] M. Jain, J. I. Choi, T. Kim, D. Bharadia, S. Seth, K. Srinivasan, P. Levis, S. Katti, and P. Sinha, "Practical, real-time, full duplex wireless," in Proceedings of the 17th annual international conference on Mobile computing and networking, ser. MobiCom '11. New York, NY, USA: ACM, 2011, pp. 301-312.

[4] E. Everett, M. Duarte, C. Dick, and A. Sabharwal, "Empowering fullduplex wireless communication by exploiting directional diversity," in Signals, Systems and Computers (ASILOMAR), 2011 Conference Record of the Forty Fifth Asilomar Conference on, Nov 2011, pp. 20022006.

[5] E. Everett, A. Sahai, and A. Sabharwal, "Passive self-interference suppression for full-duplex infrastructure nodes," Wireless Communications, IEEE Transactions on, vol. PP, no. 99, pp. 1-15, 2014.

[6] D. Bharadia, E. McMilin, and S. Katti, "Full duplex radios," in Proceedings of the ACM SIGCOMM 2013 conference on SIGCOMM, ser. SIGCOMM '13. New York, NY, USA: ACM, 2013, pp. 375-386.

[7] D. Bharadia, K. R. Joshi, and S. Katti, "Full duplex backscatter," in Proceedings of the Twelfth ACM Workshop on Hot Topics in Networks, ser. HotNets-XII. New York, NY, USA: ACM, 2013, pp. 4:1-4:7.

[8] M. Duarte and A. Sabharwal, "Full-duplex wireless communications using off-the-shelf radios: Feasibility and first results," in Signals, Systems and Computers (ASILOMAR), 2010 Conference Record of the Forty Fourth Asilomar Conference on, 2010, pp. 1558-1562.

[9] M. Duarte, C. Dick, and A. Sabharwal, "Experiment-driven characterization of full-duplex wireless systems," Wireless Communications, IEEE Transactions on, vol. 11, no. 12, pp. 4296-4307, December 2012.

[10] Y. Hua, Y. Ma, P. Liang, and A. Cirik, "Breaking the barrier of transmission noise in full-duplex radio," in Military Communications Conference, MILCOM 2013 - 2013 IEEE, Nov 2013, pp. 1558-1563.

[11] B. Schubert, J. Liszewski, and W. Keusgen, "Identification of the volterra kernels of nonlinear power amplifiers," in International Conference on Communications, Circuits and Systems, 2009. ICCCAS 2009., 2009, pp. 767-771.

[12] W. Keusgen, A. Kortke, M. Peter, and R. Weiler, "A highly flexible digital radio testbed and $60 \mathrm{GHz}$ application examples," in Microwave Conference (EuMC), 2013 European, Oct 2013, pp. 740-743.

[13] "MAFR-000229 single junction surface mount circulator," M/A-COM Technology Solutions Inc., data sheet.

[14] A. Sahai, G. Patel, C. Dick, and A. Sabharwal, "On the impact of phase noise on active cancelation in wireless full-duplex," Vehicular Technology, IEEE Transactions on, vol. 62, no. 9, pp. 4494-4510, Nov 2013. 